
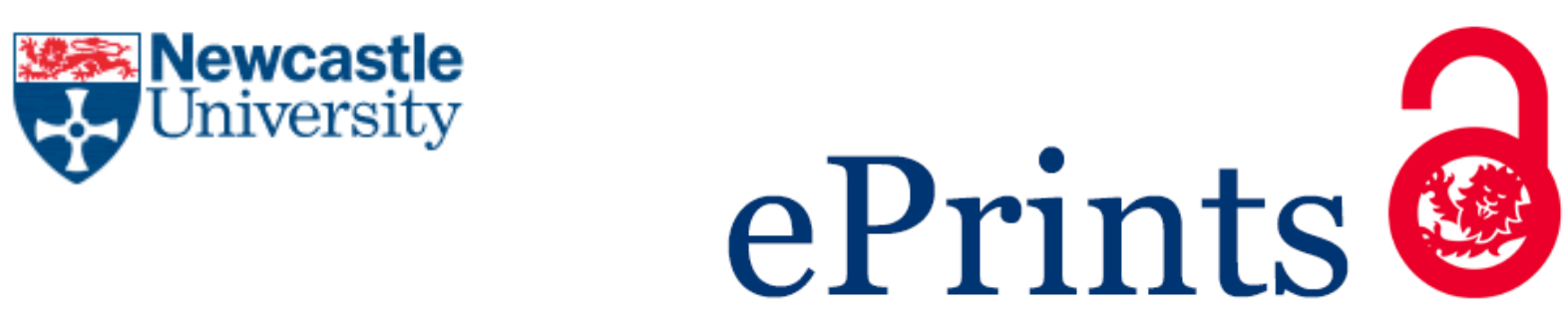

Haq I, Harris C, Taylor J, McKean MC, Brodlie M.

Should we use montelukast in wheezy children?

Archives of Disease in Childhood (2017)

DOI: https://doi.org/10.1136/archdischild-2017-312655

\title{
Copyright:
}

This article has been accepted for publication in Archives of Disease in Childhood following peer review. The definitive copyedited, typeset version is available online at:

https://doi.org/10.1136/archdischild-2017-312655

Date deposited:

$09 / 08 / 2017$ 


\section{Should we use montelukast in wheezy children?}

Iram Haq ${ }^{1,2 \#}$, Caroline Harris ${ }^{2 \#}$, Jake Taylor ${ }^{3 \#}$, Michael C McKean² and Malcolm Brodlie ${ }^{1,2^{*}}$

${ }^{1}$ Institute of Cellular Medicine, Newcastle University

2Paediatric Respiratory Medicine, Great North Children's Hospital, Newcastle upon Tyne Hospitals NHS Foundation Trust

${ }^{3}$ Medical School, Newcastle University

\#Equal contributions made

"Corresponding author: Malcolm Brodlie, MRC Clinician Scientist/Clinical Senior Lecturer, Honorary Consultant in Paediatric Respiratory Medicine. Level 3, Clinical Resource Building, Great North Children's Hospital, Queen Victoria Road, Newcastle upon Tyne, NE1 4LP. United Kingdom.

Telephone: 01912825454

E-mail: malcolm.brodlie@ncl.ac.uk

Key words: asthma; montelukast; wheeze; children; leukotriene receptor antagonist

Word count: 1246 (including suggested subheadings) 
The scale of the problem

Children who wheeze represent a major public health issue and an ongoing clinical challenge in paediatrics. Around a third of all pre-school children experience at least one episode of wheeze and $10 \%$ of school-aged children in the United Kingdom are prescribed asthma medication. Pertinently, difficult to control or severe asthma in individual children is still associated with substantial morbidity and sometimes preventable mortality on an unacceptable number of occasions in well-developed healthcare systems.

It is imperative that we manage children in the most effective way possible. In the broadest sense this starts with the accurate diagnosis of wheeze, something in itself demonstrated to be not as straightforward as perhaps we would like to imagine. Then optimal treatment and educational strategies are required to prevent or minimise the severity of future episodes. Accurate phenotyping is necessary to determine the best treatment strategy as the causes and pathogenic mechanisms of wheezing in children are multifactorial. So, where does montelukast fit in to this model of care that we aspire to deliver?

Scientific rationale for the use of montelukast and possible adverse effects

Most would agree that the science underpinning leukotriene receptor antagonists (LTRAs) is intuitively sound. LTRAs block cysteinyl leukotriene receptors that are expressed on the surface of a range of effector cells known to be pivotal in the 
pathophysiology of wheeze. Leukotrienes are pro-inflammatory lipid mediators, principally released by mast cells, that trigger bronchoconstriction, eosinophil chemotaxis and mucus secretion in the airway. LTRAs are free of many of the adverse effects associated with (oral) corticosteroids in children. A daily tablet that may be chewable is also attractive to many families. It is important to note however that behaviour change is well-recognised, which may be significant, along with very rare reports of Churg-Strauss syndrome.

Clinical use of montelukast and the concept of different phenotypes in children who wheeze

The translation of science from bench to bedside has not been completely straightforward however. The initial theoretical promise of LTRAs in children who wheeze has not been fulfilled and in "real life" terms, although some individual children experience clear benefit, treatment response in the majority often appears modest at best. However paediatric asthma guidelines are unanimous in their inclusion of LTRAs. At risk of over simplification, guidelines draw a distinction between pre-school children and those over 5; in addition, most clinicians recognise the broad phenotypes of younger children who only wheeze in association with viral respiratory tract infections, so-called episodic viral wheeze (EVW), and children with atopy and multiple-trigger wheeze (MTW) who tend to be older.

Current BTS/SIGN guidelines for pre-school children advise that LTRAs be used as first-line add on preventer therapy after low dose ICS or as alternative monotherapy 
where ICS are not tolerated. ${ }^{1}$ However, the distinction between EVW and MTW management is not entirely clear in recent practice recommendations. For children over 5, LTRA use is recommended as second-line add-on therapy where control is inadequate despite combined treatment with a long-acting beta-2 agonist (LABA) and higher-dose ICS - a point where specialist referral may be required. ${ }^{1}$

In pre-school children with EVW, montelukast has been evaluated in randomised controlled trials (RCTs) both as maintenance (preventer) and episodic (symptomatic) treatment. Individual RCTs varied in methodology, but in summary some subtle clinical benefit has been demonstrated from episodic montelukast use with reduction in health resource utilisation by around a third (OR $0.65,95 \% \mathrm{Cl} 0.47-0.89$ ) compared to placebo, ${ }^{2}$ and of reduced severity in symptoms ${ }^{3}$ (respiratory distress and disruption of activity), again by about a third. ${ }^{2}{ }^{3}$ As maintenance therapy the PREVIA study found a statistically significant reduction in exacerbation rates, by around a third (1.60 versus 2.34 episodes/year). ${ }^{4}$ In another large RCT maintenance montelukast was not associated with a reduced number of acute episodes however but there was a slight reduction in symptom scores. ${ }^{5}$ A Cochrane review did not find evidence to support maintenance or episodic montelukast in children with EVW for the primary review outcome of reduction in requirement for rescue oral corticosteroids. ${ }^{6}$

Most recently the WAIT trial randomised 1358 pre-school children with two or more previous episodes to receive montelukast or placebo at the onset of wheeze. ${ }^{7}$ Findings for the primary outcome of unscheduled medical attendances for wheeze were negative. However, in a pre-defined subgroup of children with a 5/5 
polymorphism in the ALOX5 promoter gene, which is involved in arachidonic acid metabolism, there was some benefit demonstrated (2.0 versus 2.4 unscheduled attendances/year; IRR 0.80, 95\% CI 0.68-0.95; $\mathrm{p}=0.01) .^{7}$

Montelukast has also been studied in young children after respiratory syncytial virus bronchiolitis. A large RCT found no difference in respiratory symptoms, including wheeze, in the montelukast group versus placebo. ${ }^{8}$

Although studies are limited in pre-school children with MTW, an RCT of 689 children comparing montelukast to placebo showed statistically significant, but arguably clinically modest, improvements in symptom scores by day and night, requirement for bronchodilators and oral corticosteroids and symptom-free days. ${ }^{9}$ Smaller studies have also shown reductions in bronchoconstrictive response to coldtriggered symptoms and airway hyper-responsiveness following montelukast. It is important to note however that there is clear evidence for superior efficacy of ICS over LTRAs as monotherapy for children with MTW confirming their respective positions in guidelines. ${ }^{10}$

In terms of LTRAs as add-on therapy in children evidence is limited, partly due to a shortage of good quality studies. A Cochrane review including 4 studies involving children aged 6-18 years found no significant difference in exacerbation rates between ICS and LTRA combination treatment and ICS alone at the same or increased dose. ${ }^{11}$ The BADGER trial randomised children aged 6-17 years with poorly controlled asthma on fluticasone $200 \mathrm{mcg} /$ day to receive add-on treatment in varying sequence in the form of a LABA (salmeterol), montelukast or increased 
fluticasone dose. ${ }^{12} \mathrm{~A}$ beneficial response to salmeterol was most likely compared to montelukast (relative probability $1.6,95 \% \mathrm{Cl} 1.1-2.3, \mathrm{p}=0.004$ ) or increased ICS dose (relative probability $1.7,95 \% \mathrm{Cl} 1.2-2.4, \mathrm{p}=0.005) .{ }^{12}$ Importantly, there was variation in response and some individual children responded best to LTRA or increased ICS dose.

In pursuit of precision medicine, a pragmatic way forward

One explanation for the varied results of studies discussed above is the increasingly recognised complex and dynamic heterogeneity of different endotypes in children who wheeze. This concept resonates with experiences of healthcare professionals who frequently manage children who wheeze and observe varying treatment responses and individual trajectories over time and with those who wrestle with the careful design of studies to objectively measure the efficacy of therapeutic interventions or who study the complex inter-related pathways and mechanisms involved in the pathophysiology of airway disease.

A major challenge is to successfully identify practical biomarkers or other tools to accurately, cost-effectively and rapidly select the right treatment for an individual child at the right time. Arguably the ALOX5 polymorphism subgroup in the WAIT study may provide a glimpse of this but genome sequencing is not yet a practical option at the clinical coal-face. In the absence of these key tools for precision medicine the most appropriate way forward for the thoughtful clinician is to perform an ' $n$ of 1 ' therapeutic trial in an individual patient to assess potential benefit from 
montelukast. Such a trial should be as objective as possible and finite in length remembering the variable natural history of children who wheeze. Undoubtedly a significant minority of children will benefit from montelukast and this approach would appear the most effective way to identify such children, while minimising needless over-prescription to children who do not benefit. 
Funders: MB funded by Medical Research Council Clinician Scientist fellowship (MR/M008797/1).

Competing interests: MB reports outside the submitted work investigator-led grants from Pfizer and Roche Diagnostics and personal fees paid to Newcastle University from Novartis. No other authors have any competing interests.

Contributorship statement: $\mathrm{IH}, \mathrm{CH}$ and JT: Performed literature reviews and wrote draft sections, all contributed to and approved the final version. MCM: Commented and approved the final version. MB: Drafted the initial version and completed the final version. 


\section{References}

1. BTS/SIGN. British guideline on the management of asthma: BTS/SIGN, 2016.

2. Robertson CF, Price D, Henry R, et al. Short-course montelukast for intermittent asthma in children: a randomized controlled trial. Am J Respir Crit Care Med 2007;175(4):323-9. doi: 10.1164/rccm.200510-1546OC

3. Bacharier LB, Phillips BR, Zeiger RS, et al. Episodic use of an inhaled corticosteroid or leukotriene receptor antagonist in preschool children with moderate-to-severe intermittent wheezing. J Allergy Clin Immunol 2008;122(6):1127-35 e8. doi: 10.1016/j.jaci.2008.09.029

4. Bisgaard H, Zielen S, Garcia-Garcia ML, et al. Montelukast reduces asthma exacerbations in 2- to 5-year-old children with intermittent asthma. Am J Respir Crit Care Med 2005;171(4):315-22. doi: 10.1164/rccm.200407-894OC

5. Valovirta E, Boza ML, Robertson CF, et al. Intermittent or daily montelukast versus placebo for episodic asthma in children. Ann Allergy Asthma Immunol 2011;106(6):518-26. doi: 10.1016/j.anai.2011.01.017

6. Brodlie M, Gupta A, Rodriguez-Martinez CE, et al. Leukotriene receptor antagonists as maintenance and intermittent therapy for episodic viral wheeze in children. Cochrane Database Syst Rev 2015(10):CD008202. doi: 10.1002/14651858.CD008202.pub2

7. Nwokoro $\mathrm{C}$, Pandya $\mathrm{H}$, Turner $\mathrm{S}$, et al. Intermittent montelukast in children aged 10 months to 5 years with wheeze (WAIT trial): a multicentre, randomised, placebo-controlled trial. Lancet Respir Med 2014;2(10):796-803. doi: 10.1016/S2213-2600(14)70186-9

8. Bisgaard H, Flores-Nunez A, Goh A, et al. Study of montelukast for the treatment of respiratory symptoms of post-respiratory syncytial virus bronchiolitis in 
children. Am J Respir Crit Care Med 2008;178(8):854-60. doi:

$10.1164 / \mathrm{rccm} .200706-9100 \mathrm{C}$

9. Knorr B, Franchi LM, Bisgaard H, et al. Montelukast, a leukotriene receptor antagonist, for the treatment of persistent asthma in children aged 2 to 5 years. Pediatrics 2001;108(3):E48.

10. Chauhan BF, Ducharme FM. Anti-leukotriene agents compared to inhaled corticosteroids in the management of recurrent and/or chronic asthma in adults and children. Cochrane Database Syst Rev 2012(5):CD002314. doi: 10.1002/14651858.CD002314.pub3

11. Chauhan BF, Ben Salah R, Ducharme FM. Addition of anti-leukotriene agents to inhaled corticosteroids in children with persistent asthma. Cochrane Database Syst Rev 2013(10):CD009585. doi: 10.1002/14651858.CD009585.pub2

12. Lemanske RF, Jr., Mauger DT, Sorkness CA, et al. Step-up therapy for children with uncontrolled asthma receiving inhaled corticosteroids. $N$ Engl J Med 2010;362(11):975-85. doi: 10.1056/NEJMoa1001278 